\title{
PRÁTICA PARA O APRENDIZADO DE UMA SEGUNDA LÍNGUA: NÃO COLOQUE TUDO NO MESMO BALAIO ${ }^{1}$
}

\section{Practice for Second Language Learning: Don't Throw out the Baby with the}

\section{Bathwater}

Robert DEKEYSER, Universidade de Maryland - EUA Tradução de Paula Garcia de FREITAS, Universidade Federal do Paraná

\section{APRESENTAÇÃO}

Robert DeKeyser é professor de "Aquisição de Segunda Língua" na escola de linguas, literaturas e culturas da Universidade de Maryland (EUA). O autor se ocupa de pesquisa na área de linguística aquisicional, com particular atenção para os aspectos cognitivos envolvidos na aprendizagem de uma segunda língua. Neste artigo, originalmente publicado em inglês no volume 10(1) do International Journal of English Studies em 2010 (p. 155-165), Dekeyser discute a noção de prática no ensino de línguas com base na teoria de aquisição de habilidades. De acordo com essa teoria, a aprendizagem de qualquer habilidade, seja ela cognitiva ou psicomotora, passa por dois estágios: o declarativo e o procedimentalizado. O estágio declarativo envolve o conhecimento sobre a habilidade, isto é, o conhecimento das regras e de informações sobre ela. O estágio procedimentalizado, por sua vez, compreende o conhecimento sobre como executar a habilidade. O processo de procedimentalização visa a fortalecer as produções interpretativas do conhecimento declarativo e torná-las de fácil acesso, isto é, automatizadas. A automatização do conhecimento, de acordo com a teoria, é decorrente da prática, da repetição.

Paula Garcia de Freitas

\footnotetext{
${ }^{1}$ Agradecemos ao autor e aos editores da revista por autorizarem a tradução deste artigo para o português.
} 


\section{DOSSIÊ ESPECIAL: DIDÁTICA SEM FRONTEIRAS \\ CHEREM, RAMMÉ, PEDRA \& OLMO (orgs.)}

Revista X, vol.2, 2014

RESUMO: Depois de apresentar alguns dos principais argumentos contra certas concepções restritivas de prática para a aprendizagem de uma segunda língua, este artigo reforça a validade de entender o conceito de prática de uma maneira mais ampla, ainda focada na forma, ou até mesmo nas formas, mas com a devida atenção para a relação entre forma e significado, bem como para uma sequência adequada de atividades que assegurem a aquisição do conhecimento declarativo, primeiramente, seguido da sua procedimentalização e automatização (pelo menos parcial). É feita uma breve síntese de atividades que se enquadram nesse conceito, assim como são dadas algumas recomendações para a sua adaptação às diferenças individuais dos alunos e às de grupo.

PALAVRAS-CHAVE: prática; aquisição de habilidade; desenho de currículo; correção; diferenças individuais.

ABSTRACT: After presenting some of the main arguments against certain narrow concepts of second language practice, this article argues that a broader concept of practice, still focused on form or even forms, but with due attention to form-meaning links and with appropriate sequencing of activities to ensure declarative knowledge first, followed by its proceduralization and (at least partial) automatization, is as relevant as ever. It is given a brief overview of the range of activities that fall within the scope of this concept, and recommendations are made for the adaptation of students' individual and group differences.

KEYWORDS: practice; skill acquisition; curriculum design; feedback; individual differences.

\section{PANORAMA}

A noção de prática pode ser consensual para a maioria dos professores e dos alunos, mas há algumas décadas vem suscitando discussão entre os pesquisadores da Linguística Aplicada. Krashen alegou que "a aprendizagem não se torna aquisição" (1982, p. 83), R. Ellis constatou que "os resultados [das pesquisas empíricas] não são muito animadores para a prática" (1994) e VanPatten argumentou que a força motriz para a aprendizagem linguística é a prática baseada no insumo significativo e não a prática deliberada de produção, porque "a aquisição é um subproduto da compreensão" (2003, p. 26). Talvez, nos dias de hoje, seja mais promissora a visão de Long de que a prática por meio de tarefas com foco na forma deve tomar o lugar das práticas com foco naS formaS ${ }^{2}$ e que "o maior número possível de problemas deve ser tratado por meio de aulas comunicativas que chamem a atenção dos alunos para alguns itens como e quando

\footnotetext{
${ }^{2}$ N. T. Long e Robinson (1998) chamaram de Foco na Forma a mudança ocasional da atenção para as características do código linguístico quando há algum problema de compreensão ou produção. Este conceito difere-se do que os autores chamaram de Foco naS FormaS, um tipo de ensino marcado pela conscientização das estruturas linguísticas da LE/L2, ensinadas uma por vez, configurando uma visão sintética de ensino.
} 


\section{DOSSIÊ ESPECIAL: DIDÁTICA SEM FRONTEIRAS \\ CHEREM, RAMMÉ, PEDRA \& OLMO (orgs.)}

Revista X, vol.2, 2014

os problemas surgirem” (2009, p. 384). Com base nessas poucas citações, fica claro que são muitos os argumentos contra a prática como "exercícios assassinos"3 do método audiolingual.

A pergunta que se faz é: existe ainda espaço para prática como "atividade específica para o engajamento sistemático e deliberado na segunda língua, com o objetivo de desenvolver conhecimentos e habilidades sobre ela" (DEKEYSER, 2007a, p. 8)? Parece que a resposta para essa questão depende dos objetivos para a prática, se eles valem à pena e são realizáveis, ou, mais precisamente, se são mais viáveis através da prática, isto é, da repetição ou por outros meios. Vamos argumentar aqui que a resposta para essa pergunta é afirmativa, e que as atividades para a prática podem assumir muitas formas, cuja eficácia depende dos alunos e do contexto.

\section{RAZÕES PARA REJEITAR UM CONCEITO LIMITADO DE PRÁTICA SISTEMÁTICA}

Ao se falar sobre prática, muitas vezes vem à mente o estereótipo de uma sala de aula audiolingual, onde os alunos repetem e transformam frases até cansarem. Muito já foi argumentado que tais exercícios são limitados, pois não ensinam aos alunos como desenvolver e expressar o comportamento-alvo de conceptualização do significado por meio da língua, muito menos permitem fazê-lo de forma criativa (vide, por exemplo, DEKEYSER, 1998; KRASHEN, 1982, 1985).

No entanto, a crítica à prática sistemática é muito mais profunda. Alguns autores, embora não rejeitando completamente o foco na forma como o faz Krashen, colocam em dúvida a utilidade da prática repetitiva de produção. Um problema central a este respeito é que esse tipo de prática não necessariamente faz com que os alunos exercitem o comportamento-alvo, no sentido do processamento desejado das conexões do significado para a forma, mas tenta ensinar o processamento pelo caminho inverso, isto é, das formas para o significado, por meio do insumo compreensível (vide VANPATTEN, 2004; WONG; VANPATTEN, 2003).

\footnotetext{
${ }^{3}$ N. T. O método audiolingual foi chamado de "exercícios assassinos" (drill and kill) por muitos autores que julgavam que uma aula inteira de repetição de modelos era capaz de matar qualquer interesse do aluno pela aprendizagem da língua (cf. LEFFA, 2006).
} 


\section{DOSSIÊ ESPECIAL: DIDÁTICA SEM FRONTEIRAS \\ CHEREM, RAMMÉ, PEDRA \& OLMO (orgs.)}

Revista X, vol.2, 2014

Há autores que vão ainda mais longe e rejeitam qualquer ensino ou prática focada na forma ${ }^{4}$, porque nenhuma atividade elaborada em torno de uma forma específica é tão eficaz para a aquisição do que as tarefas da vida real, que permitem um foco mais incidental sobre as estruturas da língua (vide LONG, 1991; LONG; ROBINSON, 1998), dado que durante essas atividades o aluno “... está imbuído do papel da troca, por isso está motivado a aprender o que é necessário e esperado para a sua resposta, já sabe o significado do que ele está tentando expressar, então libera recursos atencionais para a estrutura presente na resposta e ouve a forma correta em estreita justaposição com o que espera, facilitando a comparação cognitiva" (LONG, 2009, p. 385).

Outros, ainda, não duvidam da utilidade da prática focada nas formas, mas acham que ela deva ser de uso limitado. Na visão desses autores, a repetição não ajuda na aquisição de novas estruturas gramaticais, mas pode contribuir para a automatização do conhecimento já existente (R. ELLIS, 1993).

\section{RAZÕES PARA APOIAR UM CONCEITO AMPLO DE PRÁTICA SISTEMÁTICA}

Deve-se pensar com cuidado, no entanto, antes de concluir que qualquer prática sistemática (de compreensão ou de produção) das formas seja inútil. Em primeiro lugar, desenvolver a habilidade de uso das formas, além da mera familiaridade com elas, é certamente um dos objetivos do ensino de línguas, e não há razão para supor que a teoria de aquisição de habilidades, que enfatiza o papel do conhecimento declarativo no desenvolvimento do conhecimento procedimentalizado e eventualmente automatizado, que tem sido aplicada a uma variedade de domínios cognitivos, (vide, por exemplo, ANDERSON; LEBIERE, 1998; ANDERSON et al, 2004), não possa ser aplicada também à aprendizagem linguística. Como argumentou DeKeyser (2007b), os resultados oriundos da teoria de aquisição de segunda língua são mais compatíveis com este ponto de vista do que é frequentemente admitido, e grande parte do mal-entendido se baseia em uma interpretação muito limitada de "um tipo de conhecimento se

\footnotetext{
${ }^{4}$ N. T. O termo forma refere-se aos traços de superfície de uma língua (VANPATTEN, 2002), como morfemas, elementos sintáticos, marcadores discursivos, etc. e as regras para o seu uso. É o mesmo que estrutura linguística.
} 


\section{DOSSIÊ ESPECIAL: DIDÁTICA SEM FRONTEIRAS \\ CHEREM, RAMMÉ, PEDRA \& OLMO (orgs.)}

Revista X, vol.2, 2014

transformando em outro ao invés de um tipo de conhecimento que desempenha um papel causal para o desenvolvimento do outro" (DEKEYSER, 2009; DEKEYSER; CRIADO-SÁNCHEZ, 2013).

Em outras palavras, com o aumento da prática e da proficiência, não são apenas as representações linguísticas que mudam, mas também as habilidades para usá-las (KROLL; LINCK, 2007). A recuperação do conhecimento ao longo do processamento da língua é uma habilidade complexa que requer muita prática, e o conceito aplica-se ao léxico, bem como à gramática, independentemente do modo como se vê o processo de automatização, isto é, como um uso sempre mais fluente das regras ou como o processo gradual de recuperação que substitui mais e mais o uso da regra, como Kroll e Linck parecem defender seguindo a teoria de automatização de Logan (1988, 1992). Segalowitz (2010) apresenta um relato teórico completo de como esses processos recuperação e automatização - estão no centro do desenvolvimento da fluência, e Nation e Newton (2009) fazem um elenco sistemático de recomendações sobre como desenvolver a fluência na compreensão e na produção oral por meio de planejamento, prepaaração, repetição, restrição do tempo para a resposta e do uso de itens com os quais os alunos já estão familiarizados.

Talvez o mais importante no âmbito das pesquisas sobre aquisição de segunda língua, e que muitos autores têm argumentado, é que, para qualquer língua, é difícil ou quase impossível aprender o grande número de elementos que a constituem por meio da simples exposição e da "prática" com o objetivo único de se comunicar nela, porque esses elementos têm baixa frequência ou pouca relevância, especialmente quando a prática se concentra no mapeamento forma-significado (vide DEKEYSER, 2005; PICA, 2009). O insumo destacado ${ }^{5}$ e o ensino explícito da forma podem solucionar esse problema em alguns casos, mas em outros, é necessário realizar ainda a prática excessiva para a procedimentalização do mapeamento entre forma e significado para a produção e/ ou compreensão da língua-alvo. Particularmente, esse é o caso quando novas distinções semânticas devem ser adquiridas (por exemplo, as diferenças aspectuais para a conjugação dos verbos em línguas românicas ou a distinção de especificidade +/- para o artigo em inglês), ou quando certos significados ou

5 N. T. Insumo destacado é uma estratégia de ensino em que a forma linguística é destacada tipograficamente no texto escrito (REINDERS; ELLIS, 2009) ou destacada na entonação, no volume de voz ou em pausas antes/ depois da aparição da estrutura-alvo em um texto oral (REINDERS; CHO, 2011). 


\section{DOSSIÊ ESPECIAL: DIDÁTICA SEM FRONTEIRAS \\ CHEREM, RAMMÉ, PEDRA \& OLMO (orgs.)}

Revista X, vol.2, 2014

combinações de significados da L2 precisam ser explicados aos alunos por não terem correspondentes explícitos na gramática da L1 (como a marca de plural da língua inglesa para chineses, japoneses e coreanos). A procedimentalização e a automatização (parcial, pelo menos) dessas distinções precisa de uma grande quantidade de prática.

Então, Nation e Newton (2010) podem muito bem estar certos ao dizerem que "o foco na forma não deve ultrapassar $25 \%$ de toda a gama de contato que os alunos têm com a língua" (p. 148), dependendo da situação. Se os alunos têm muito contato significativo com a língua fora da sala de aula, esse percentual pode ser maior; caso o contato dos alunos se limite ao contexto escolar, então o percentual de foco sobre as estruturas linguísticas deve ser inferior aos $25 \%$. Por outro lado, como o livro desses autores deixa claro, atividades com foco no significado podem e devem ainda fornecer um pouco de foco na forma; para desenvolver a fluência, por exemplo, os autores propõem atividades que incluem até mesmo a repetição de frases e expressões memorizadas.

É claro que nenhum grau de ensino e de prática de sala de aula vai levar a um perfeito conhecimento da língua; seria totalmente ingênuo esperar por isso, uma vez que até mesmo muitos anos de residência no país onde se fala a língua-alvo não garantem tal excelência (ABRAHAMSSON; HYLTENSTAM, 2009; DEKEYSER; ALFISHABTAY; RAVID, 2010; DEKEYSER; LARSON-HALL, 2005; HYLTENSTAM; ABRAHAMSSON, 2003). Concordamos, portanto, que a "prática não leva à perfeição", mas é necessária para o ensino (e para a aprendizagem) de uma língua: “a prática comunicativa (...) não é suficiente para levar os alunos a altos níveis de fluência e precisão de todos os elementos da aquisição de segunda língua", mas "quando a prática é definida como uma oportunidade para o uso significativo da língua e para a prática reflexiva e vigorosa de aspectos linguísticos difíceis, então o papel da prática é claramente benéfico e até mesmo essencial” (LIGHTBOWN, 2000, p. 443).

\section{PRÁTICA SISTEMÁTICA É “UMA CASA COM MUITOS QUARTOS”}

Deve ficar claro, a partir da discussão acima, que o termo "prática", ou até mesmo "prática sistemática", abarca muito mais do que os exercícios mecanizados. Mesmo os "exercícios mecanizados", no sentido de produção repetida de certas formas, não se limitam à noção de agir como papagaio. Paulston e Bruder (1976) já haviam 


\section{DOSSIÊ ESPECIAL: DIDÁTICA SEM FRONTEIRAS \\ CHEREM, RAMMÉ, PEDRA \& OLMO (orgs.)}

Revista X, vol.2, 2014

distinguido os exercícios mecanizados dos exercícios significativos e dos comunicativos $^{6}$, e outros pesquisadores fizeram distinções semelhantes (vide DEKEYSER; CRIADO-SÁNCHEZ, 2013b). Exercícios comunicativos desenvolvem nos alunos o comportamento-alvo de ligar uma forma a um significado, e são, portanto, úteis nesse sentido (WONG; VANPATTEN, 2003).

Claro que, por razões cognitivas e afetivas (incorporando estruturas em discursos mais realistas nos quais o foco sobre a forma não é tão óbvio e fornecendo atividades mais agradáveis e com maior validade), os professores devem ir, o mais rápido possível, além da prática estritamente focada na forma. Vários tipos de role play ou atividades com lacunas de informação (information-gap activities) podem ser escolhidos de modo a permitir o uso essencial, ou pelo menos bastante provável, de certas estruturas problemáticas. (cf. os conceitos de essencialidade da tarefa e utilidade da tarefa - taskessentialness e task-usefulness de Loschky e Bley-Vroman's, 1993). Com a participação de falantes nativos, esse tipo de atividade não só pode se tornar uma fonte de prática realista, mas também de feedback corretivo de vários tipos, em particular do tipo reformulação da fala dos alunos (recasts).

Finalmente, no outro extremo do espectro, em oposição aos exercícios, estão as abordagens de ensino por conteúdo e por tarefas, as quais não apresentam mais nenhum foco naS formaS, mas, mesmo assim, oferecem inúmeras oportunidades para o foco na forma reativo (LONG, 2009; NORRIS, 2009). Tais contextos de aprendizagem são certamente desejáveis, mas é questionável até que ponto eles podem ser criados nos estágios iniciais da aprendizagem de línguas estrangeiras, quando as ferramentas linguísticas à disposição dos alunos, tanto para a produção quanto para a compreensão são ainda bastante limitadas.

Todos esses tipos de prática, que vão muito além dos "exercícios assassinos", mas que satisfazem a nossa definição mais ampla de "prática sistemática", cumprem funções específicas no processo de aprendizagem que a mera exposição ao insumo e a interação não podem cumprir.

\footnotetext{
6 N. T. Os exercícios mecanizados são exclusivamente centrados na forma e não requerem, necessariamente, a atenção do aprendiz para o significado semântico ou funcional da estrutura durante a sua realização. Os exercícios significativos requerem o processamento/ entendimento do significado semântico ou funcional de sentenças e enunciados, sem a necessidade de se comunicar algo a alguém, como a resposta a uma pergunta retórica, por exemplo. Já os exercícios comunicativos requerem o uso da língua em contexto situado e propositado da língua, enquanto as regras, previamente ensinadas, foco da atividade, podem ser mantidas na mente" (DEKEYSER, 1998, p. 52).
} 


\section{DOSSIÊ ESPECIAL: DIDÁTICA SEM FRONTEIRAS \\ CHEREM, RAMMÉ, PEDRA \& OLMO (orgs.)}

Revista X, vol.2, 2014

\section{ADAPTANDO A PRÁTICA AOS INDIVÍDUOS E AOS CONTEXTOS}

Nem todos os tipos de prática vão funcionar igualmente bem em todas as circunstâncias. Mais óbvio, talvez, é que crianças não vão se beneficiar de certos tipos de prática que seriam mais adequados para adultos. Por outro lado, as crianças podem estar mais dispostas a passar pelos exercícios mecanizados e repetitivos do que os adultos ou que os adolescentes, porém, como já vimos, esse tipo de prática é de uso limitado. Atividades de role-play apropriadas para cada faixa etária, tarefas da vida real ou até mesmo assuntos para a conversação podem ser difíceis de serem formuladas. Em compensação, se a prática for dada na forma de um jogo, as crianças, muitas vezes, se mostram mais dispostas a se engajar do que os adultos (CAMERON, 2001; MUÑOZ, 2007).

Além da idade, a cultura precisa ser levada em consideração, tanto no sentido geral quanto no sentido da cultura escolar, isto é, das experiências anteriores dos alunos com o ensino e com a aprendizagem. Os diferentes países variam drasticamente no modo como enfatizam ou toleram a repetição, a memorização, a criatividade, a diversão, a correção negativa, a autoestima, e outros ingredientes que caracterizam diferentes tipos de atividade para a prática em diferentes graus. Esses elementos têm claras implicações na disposição do professor em adotar os diferentes tipos de prática e na vontade dos alunos em aprender com eles. (DUFF, 1995; GORSUCH, 2000).

Talvez ainda mais importante seja considerar a aptidão dos alunos em se beneficiar dos vários tipos de prática (a partir das muitas maneiras de ensinar de modo geral). A aptidão é tomada aqui no sentido mais amplo de "aptidões cognitivas", isto é, características "afetivas", tais como motivação, atitude, personalidade e até mesmo conhecimentos prévios e habilidades. Robinson (2007), em particular, oferece muitas hipóteses sobre como certos tipos de atividades atuam em aptidões específicas: a aprendizagem explícita de regras conta com um conjunto de aptidões que envolve, principalmente, a sensibilidade gramatical e a memorização; a aptidão para o foco na forma por meio correções implícitas (recasts) baseia-se na velocidade e na capacidade fonológica da memória de trabalho; a aptidão para a aprendizagem incidental da língua durante a conversação depende da capacidade de raciocínio analógico e de inferência sobre os significados das palavras; a aptidão para a aprendizagem incidental de 


\section{DOSSIÊ ESPECIAL: DIDÁTICA SEM FRONTEIRAS \\ CHEREM, RAMMÉ, PEDRA \& OLMO (orgs.)}

Revista X, vol.2, 2014

conteúdos durante a leitura é decorrente da velocidade e da capacidade de concentração da memória de trabalho para com o texto.

Os professores precisam sentir como podem adaptar as atividades de prática para seus alunos. Mesmo que a adaptação às (muitas) aptidões individuais seja dificilmente possível no contexto de sala de aula, e que talvez a sistematização do conhecimento possa ser auxiliada por atividades mais avançadas de prática por computador, é indispensável que o professor adapte atividades de prática para determinado grupo de alunos em função da idade ou da sua bagagem cultural.

\section{OUTRAS QUESTÕES}

Permanecem ainda muitas questões sobre quais tipos de prática são melhores, e, agora que o cenário teórico na Linguística Aplicada é menos hostil ao ensino e a prática de formas do que era há uma ou duas décadas atrás, um número maior de pesquisas empíricas está começando a se concentrar sobre esse tema.

Várias questões que dizem respeito à frequência ideal, ao imediatismo e à natureza das estratégias de correção deixam claro que o quadro é bastante complexo. Muitas maneiras de corrigir podem ser, ao mesmo tempo, positivas e negativas. Além disso, às vezes fica difícil determinar se a correção pode ser considerada implícita ou explícita (especialmente porque a correção dada implicitamente pode ser registrada explicitamente pelo aluno e a falta de metalinguagem durante a correção não pode ser confundida com falta de aprendizagem explícita, isto é, com a falta de consciência sobre a forma correta presente na correção). No geral, a literatura é favorável à correção do tipo "reformulação da fala do aluno" (recasts), porque ela fornece evidências positivas num momento em que o aluno está prontamente receptivo a elas (LONG, 2009; PICA, 2009). A eficácia dessa estratégia de correção depende, no entanto, da forma linguística que está sendo corrigida, dos conhecimentos prévios, da vontade do aluno em focar sobre a forma e das suas aptidões (vide EGI, 2007; MACKEY et al 2002; RANTA; LYSTER, 2007; ROBINSON, 2007; SACHS; SUH, 2007; SAGARRA, 2007; SHEEN, 2006, 2007; TROFIMOVICH et al, 2007). Para excelentes revisões de literatura e metaanálises referentes às correções em geral e, especialmente, sobre a correção por meio da reformulação da fala do aluno (recasts), leia Leeman (2007), Li (2010), Long (2007), Lyster e Saito (2010) e Russell e Spada (2006). 


\section{DOSSIÊ ESPECIAL: DIDÁTICA SEM FRONTEIRAS \\ CHEREM, RAMMÉ, PEDRA \& OLMO (orgs.)}

Revista X, vol.2, 2014

Uma outra grande questão se refere à quantidade de prática ao longo do tempo. No âmbito da Psicologia da Educação, muitas vezes foi documentado que a distribuição de uma determinada quantidade de prática por períodos longos é benéfica (SEABROOK et al, 2005). No entanto, Collins et al (1999) e Serrano (2011) mostram que há limites para quão longa deve ser essa distribuição, pois estendê-la por anos pode ser pior do que concentrá-la em poucos meses, ou ainda, levar mais de um ano para praticar algo que poderia ser beneficiado por apenas um semestre, não ajuda na aprendizagem.

Não pode ser negligenciada, ainda, a questão de como um conhecimento declarativo inicial deve ser transmitido aos alunos. Para que os alunos possam praticar proficuamente uma determinada forma, é necessário que haja conhecimento sobre ela a ser praticado, e a vivência da sala de aula tem mostrado cada vez mais que a apresentação tradicional de regras gramaticais por parte do professor não é a única maneira de se conseguir isso. Mesmo os defensores de abordagens muito distintas, como os cognitivistas que adotam abordagens baseadas em tarefas (ELLIS, 2003; LONG, 2009) e os socioculturais, que advogam por um conhecimento socialmente gerado (SWAIN; LAPKIN, 1998), podem concordar que o conhecimento explícito é mais bem retido na memória de trabalho quando ele é oferecido em resposta às perguntas que surgem a partir de interações comunicativas. No entanto, como fazer para que esse processo seja maximamente eficiente e como garantir que esse conhecimento seja especialmente codificado para poder ser transferido para outras tarefas ainda continuam sendo questões difíceis de serem respondidas.

A questão mais importante de todas, no entanto, é como as aptidões dos alunos, as características das formas a serem adquiridas e as diferentes modalidades de prática interagem para determinar o aprendizado. Como afirmado acima, já há evidências de que a eficácia das várias formas de prática e de correção depende das aptidões dos alunos e das características da forma a ser aprendida, mas, até agora, nenhuma pesquisa abordou a interação entre as três variáveis. Além disso, na área de Linguística Aplicada, não existe nenhuma pesquisa sobre a interação da prática com a natureza exata do conhecimento dos alunos em termos de aquisição de habilidades. Esse tipo de pesquisa não é possível nas salas de aula tradicionais, mas em contextos assistidos por computadores, estudos envolvendo modelos de alunos e correções adaptativas já apontam um caminho, julgando pelo trabalho em outras áreas, como álgebra, ciência da computação ou física. (vide KOEDINGER; CORBETT, 2006; LESGOLD, 2003). 


\section{DOSSIÊ ESPECIAL: DIDÁTICA SEM FRONTEIRAS \\ CHEREM, RAMMÉ, PEDRA \& OLMO (orgs.)}

Revista X, vol.2, 2014

Ao mesmo tempo, no entanto, é importante não perder de foco a sala de aula como um lugar potencial para a investigação. A sala de aula não só é necessária para maximizar a validade ecológica das pesquisas, como também é o único jeito de assegurar estudos longitudinais sobre o ensino de línguas (PICA, 2009) e sobre os efeitos de diferentes tipos de ensino e de prática para determinadas estruturas linguísticas. Isso exigirá, entretanto, que os pesquisadores que realizam estudos em sala de aula se esforcem para projetar experimentos controlados ao invés de simplesmente descrevê-los ou relacioná-los com outras pesquisas. Além disso, os pesquisadores precisam ser mais orientados psicolinguisticamente para compreender que um estudo que lida diretamente com os processos que mais importam para a maioria dos alunos e professores de segunda língua não pode ser $100 \%$ controlado, mas deve ser conduzido da maneira mais controlada possível.

\section{CONCLUSÃO}

A aquisição de uma segunda língua requer grandes quantidades de prática, como qualquer outro tipo de habilidade. A mera exposição ou a interação pouco estruturada na língua-alvo não podem ser consideradas como uma prática ideal (com base na teoria de aquisição de habilidades). Mais atividades para a prática sistemática são necessárias, e isso não significa a repetição manipulada de uma forma, mas sim que pode acontecer de várias maneiras, desde exercícios comunicativos ao início do processo de aprendizagem até atividades de role-play, tarefas comunicativas ou pelo ensino por conteúdo. A prática, como qualquer outro aspecto do currículo, deve ser adaptada às características dos alunos e ao contexto de ensino. Pesquisas sobre como otimizar essa adaptação podem ser um ramo intelectualmente estimulante para os próximos anos, com resultados altamente confiáveis e praticáveis. 


\section{DOSSIÊ ESPECIAL: DIDÁTICA SEM FRONTEIRAS \\ CHEREM, RAMMÉ, PEDRA \& OLMO (orgs.)}

Revista X, vol.2, 2014

\section{REFERÊNCIAS BIBLIOGRÁFICAS}

ABRAHAMSSON, N.; HYLTENSTAM, K. Age of onset and nativelikeness in a second language: Listener perception versus linguistic scrutiny. Language Learning, v. 59, n. 2, pp. 249-306, 2009.

ANDERSON, J. R., BOTHELL, D., BYRNE, M. D., DOUGLASS, S., LEBIERE, C., QIN, Y. An integrated theory of the mind. Psychological Review, v. 111, n. 4, pp. 1036-1060, 2004.

ANDERSON, J. R., LEBIERE, C. The atomic components of thought. Mahwah: Lawrence Erlbaum, 1998.

CAMERON, L. Teaching languages to young learners. Cambridge: Cambridge University Press, 2001.

COLlinS, L., HALTER, R. H., LIGHTBOWN, P. M., SPADA, N. Time and the distribution of time in L2 instruction. TESOL Quarterly, v. 33, n. 4, pp. 655-680, 1999.

DEKEYSER, R. M. Beyond focus on form: Cognitive perspectives on learning and practicing second language grammar. In Doughty C., Williams, J. (Eds.), Focus on form in classroom second language acquisition. New York: Cambridge University Press, 1998, pp. 42-63.

M. Cognitive-psychological processes in second language learning. In: LONG, M.; DOUGHTY, C. (Eds.), Handbook of Second Language Teaching. Oxford, UK: Wiley-Blackwell, 2009, pp. 119-138.

Situating the concept of practice. In DEKEYSER, R. M. (Ed.), Practice in a second language: Perspectives from applied linguistics and cognitive psychology, New York: Cambridge University Press. 2007a, pp. 1-18.

Skill acquisition theory. In VANPATTEN, B.; WILLIAMS, J. (Eds.), Theories in Second Language Acquisition: An introduction. Mahwah: Erlbaum, 2007b, pp. 97-112.

.What makes learning second-language grammar difficult? A review of issues.

Language Learning, v. 55, n. 1, p. 1-25, 2005.

DEKEYSER, R. M., ALFI-SHABTAY, I., RAVID, D. Cross-linguistic evidence for the nature of age effects in second language acquisition. Applied Psycholinguistics, v. 31, n. 3, pp. 413-438, 2010.

DEKEYSER, R. M., CRIADO-SÁNCHEZ, R. Automatization, skill acquisition, and practice in second language acquisition. In CHAPELLE, C. A. (Ed.), The Encyclopedia of Applied Linguistics. Oxford: Wiley-Blackwell, 2013a.

Practice in second language instruction. In Chapelle, C. A. (Ed.), The

Encyclopedia of Applied Linguistics. Oxford: Wiley-Blackwell, 2013b.

DEKEYSER, R. M., LARSON-HALL, J. What does the critical period really mean? In: Kroll, J. F.; de Groot, A. M. B. (Eds.), Handbook of Bilingualism: Psycholinguistic Approaches). Oxford: Oxford University Press, 2005, pp. 89-108.

DUFF, P. A. An ethnography of communication in immersion classrooms in Hungary. TESOL Quarterly, n. 29, pp. 505-537, 2005.

EGI, T. Recasts, learners' interpretations, and L2 development. In: Mackey, A. (Ed.), Conversational interaction in second language acquisition. New York: Oxford University Press, 2007, p. 249-360.

ELLIS, R. Task-based language learning and teaching. Oxford: Oxford University Press, 2003. 


\section{DOSSIÊ ESPECIAL: DIDÁTICA SEM FRONTEIRAS \\ CHEREM, RAMMÉ, PEDRA \& OLMO (orgs.)}

Revista X, vol.2, 2014

.The structural syllabus and second language acquisition. TESOL Quarterly, v. 27, n. 1, pp. 91-113, 1993.

ELLIS, R. The study of second language acquisition. Oxford: Oxford University Press, 1994.

GORSUCH, G. J. EFL educational policies and educational cultures: Influences on teachers' approval of communicative activities. TESOL Quarterly, v. 34, n. 4, p. 675$710,2000$.

HYLTENSTAM, K., ABRAHAMSSON, N. Maturational constraints in second language acquisition. In: DOUGHTY, C. J.; LONG, M. H (Eds.), Handbook of second language acquisition. Oxford: Blackwell, 2003, p. 539-588.

KOEDINGER, K. R., CORBETT, A. Cognitive tutors: Technology bringing learning sciences to the classroom. In: SAWYER, R. K. (Ed.). The Cambridge Handbook of the Learning Sciences. New York: Cambridge University Press, 2006, p. 61-77.

KRASHEN, S. D. Principles and practice in second language acquisition. Englewood Cliffs: Prentice-Hall, 1982.

.The input hypothesis. London/New York: Longman, 1985.

KROLL, J. F., LINCK, J. A. Representation and skill in second language learners and proficiente bilinguals. In: KECSKES, I.; ALBERTAZZI, L. (Eds.), Grammatical gender in the bilingual lexicon: A psycholinguistic approach. Dordrecht: Springer, 2007, p. 237-269.

LEEMAN, J. Feedback in L2 learning: Responding to errors during practice. In: R. DEKEYSER R. M. (Ed.), Practice in a second language. Perspectives from applied linguistics and cognitive psychology. New York: Cambridge University Press, 2007, p. 111-137.

LEFFA, V. J. A aprendizagem de línguas mediada por computador. In: LEFFA, V. J. (Org.). Pesquisa em linguística aplicada: temas e métodos. Pelotas: Educat, 2006. pp. 11-36.

LESGOLD, A. (2003). Intelligent tutoring systems. In: NADEL, L. (Ed.), Encyclopedia of Cognitive Science (Vol. 2). Londres: Macmillan, 2003, pp. 593-598.

LI, S. The effectiveness of corrective feedback in SLA: A meta-analysis. Language Learning, v. 60, n. 2, p. 309-365, 2010.

LIGHTBOWN, P. Classroom SLA research and second language teaching. Applied Linguistics, v. 21, n. 4, pp. 431-462, 2000.

LONG, M. Focus on form: a design feature in language teaching methodology. In: DEBOT, K., COSTE, D.; GINSBERG, R.; KRAMSCH, C. (Eds.), Foreign language research in cross-cultural perspective. Amsterdã/Filadelfia: John Benjamins, pp. 3952, 1991.

LONG, M. H., ROBINSON, P. Focus on form: Theory, research, and practice. In: DOUGHTY, C. WILLIAMS, J. (Eds.), Focus on form in classroom second language acquisition. New York: Cambridge University Press, 1998, pp. 15-41.

LONG, M. Methodological principles for language teaching. In: LONG, M.; DOUGHTY, C. (Eds.), Handbook of second language teaching. Oxford, UK: WileyBlackwell, 2009, pp. 373-394.

LONG, M. Problems in SLA. Mahwah: Erlbaum, 2007.

LOSCHKY, L., BLEY-VROMAN, R. Grammar and task-based methodology. In: CROOKES, G.; GASS, S. (Eds.), Tasks and language learning: Integrating theory and practice, Clevedon: Multilingual Matters, 1993, pp. 122-167.

LYSTER, R., SAITO, K. Oral feedback in classroom SLA: A meta-analysis. Studies in Second Language Acquisition, v. 32, n. 2, pp. 265-302, 2010. 


\section{DOSSIÊ ESPECIAL: DIDÁTICA SEM FRONTEIRAS \\ CHEREM, RAMMÉ, PEDRA \& OLMO (orgs.)}

Revista X, vol.2, 2014

MACKEY, A., PHILP, J., EGI, T., FUJII, A., \& TATSUMI, T. Individual differences in working memory, noticing of interactional feedback and L2 development. In: ROBINSON P. (Ed.). Individual differences and instructed language learning, Amsterdam/Philadelphia: Benjamins, 2002, p.p 181-209.

MUÑOZ, C. Age differences and their implications for practice. In: DEKEYSER, R. M (Ed.). Practice in a second language: Perspectives from applied linguistics and cognitive psychology. Nova York: Cambridge University Press, 2007, pp. 229-255.

NATION, I. S. P., NEWTON, J. Teaching ESL/EFL listening and speaking. Londres: Routledge, 2009.

NORRIS, J. Task-based teaching and testing. In: LONG, M.; DOUGHTY, C. (Eds.). The Handbook of Language Teaching. Oxford: Wiley-Blackwell, 2009, pp. 578-594. PAULSTON, C. B., BRUDER, M. N. Teaching English as a second language: Techniques and Procedures. Cambridge, MA: Winthrop, 1976.

PICA, T. Second language acquisition in the instructional environment. In: RITCHIE, W.; BHATIA, T. (Eds.), The New Handbook of Second Language Acquisition. Bingley, UK: Emerald, 2009, pp. 473-501.

RANTA, L., LYSTER, R. A cognitive approach to improving immersion students' oral language abilities: The Awareness-Practice-Feedback sequence. In: DEKEYSER, R. M. (Ed.), Practice in a second language: Perspectives from Applied Linguistics and Cognitive Psychology. Nova York: Cambridge University Press, 2007, pp. 141-160.

REINDERS, H., CHO, M. Encouraging informal language learning with mobile technology: does it work? Journal of Second Language Teaching and Research, Preston, v. 1, n. 1, pp. 3-19, 2011. Disponível em:

<http://pops.uclan.ac.uk/index.php/jsltr/article/view/8/1>, Acesso em 22/07/2012.

REINDERS, H., ELLIS, R. The effects of two types of input on intake and the acquisition of implicit and explicit knowledge. In: ELLIS, R., LOEWEN, S, ELDER, C, ERLAM, R, PHILP, J.; REIDERS, H. Implicit and explicit knowledge in second Language Learning, testing and teaching. Bristol, Buffalo, Toronto: Multilingual Matters, 2009, pp. 281-302.

ROBINSON, P. (2007). Aptitudes, abilities, contexts, and practice. In: DEKEYSER, R. M. (Ed.), Practice in second language. Perspectives from applied linguistics and cognitive psychology. Cambridge, UK: Cambridge University Press, 2007, pp. 256286.

RUSSELL, J., SPADA, N. The effectiveness of corrective feedback for the acquisition of L2 grammar: A meta-analysis of the research. In: NORRIS, J.; ORTEGA, L. (Eds.), Synthesizing research on language learning and teaching. Filadelfia / Amsterdã: John Benjamins, 2006, pp. 133-164.

SACHS, R., SUH, B. R. Textually enhanced recasts, learner awareness, and L2 outcomes in synchronous computer-mediated interaction. In: Mackey, A. (Ed.), Conversational interaction in second language acquisition. Nova York: Oxford University Press, 2007, pp. 197-227.

SAGARRA, N. Computer-delivered recasts, working memory, L2 development and modified output. In: Mackey, A. (Ed.), Conversational interaction and second language acquisition. Oxford: Oxford University Press, 2007.

SEABROOK, E., BROWN, G. D. A., SOLITY, J. E. Distributed and massed practice: From laboratory to classroom. Applied Cognitive Psychology, n. 19, pp. 107-122, 2005.

SEGALOWITZ, N. Cognitive bases of second language fluency. Nova York: Routledge, 2010. 


\section{DOSSIÊ ESPECIAL: DIDÁTICA SEM FRONTEIRAS \\ CHEREM, RAMMÉ, PEDRA \& OLMO (orgs.)}

Revista X, vol.2, 2014

SERRANO, R. The time factor in EFL classroom practice. Language Learning, v. 61, n. 1, 2011.

SHEEN, Y. Exploring the Relationship between Characteristics of Recast and Learner Uptake. Language Teaching Research, v. 10, n. 4, pp. 361-392, 2006.

SHEEN, Y. The effect of focused written corrective feedback and language aptitude on ESL learners' acquisition of articles. TESOL Quarterly, v. 41, n. 2, pp. 255-283, 2007.

SWAIN, M., LAPKIN, S. Interaction and second language learning: Two adolescent French immersion students working together. The Modern Language Journal, v. 82, n. 3, pp. 320-337, 1998.

TROFIMOVICH, P., AMMAR, A., GATBONTON, E. How effective are recasts? The role of attention, memory, and analytical ability. In: MACKEY, A. (Ed.), Conversational interaction in second language acquisition. Nova York: Oxford University Press, 2007, pp. 171-195.

VANPATTEN, B. From input to output: A teacher's guide to second language acquisition. Nova York: McGraw-Hill, 2003.

Input processing in second language acquisition. In: VANPATTEN, B.

(Ed.), Processing instruction. Theory, research, and commentary. Mahwah: Erlbaum, 2004, pp. 5-31.

. Processing Instruction: an update. Language Learning, California, v. 52, n. 4, p. 755803, 2002.

WONG, W., VANPATTEN, B. The evidence is IN: Drills are OUT. Foreign Language Annals, v. 36, n. 3, pp. 403-423, 2003. 